\title{
Novel mutations in Lrp6 orthologs in mouse and human neural tube defects affect a highly dosage-sensitive Wnt non-canonical planar cell polarity pathway
}

\author{
Redouane Allache ${ }^{1, \dagger}$, Stéphanie Lachance ${ }^{1, \dagger}$, Marie Claude Guyot ${ }^{1}$, Patrizia De Marco², \\ Elisa Merello², Monica J. Justice ${ }^{3}$, Valeria Capra² and Zoha Kibar ${ }^{1, *}$ \\ ${ }^{1} \mathrm{CHU}$ Sainte Justine Research Center and University of Montréal, Montréal, QC, Canada H3T 1C5, ${ }^{2} \mathrm{U} . \mathrm{O}$. Neurochirurgia, \\ Istituto Giannina Gaslini, Genova 16147, Italy and ${ }^{3}$ Department of Molecular and Human Genetics, Baylor College of \\ Medicine, Houston, TX 77030 USA
}

Received August 10, 2013; Revised October 16, 2013; Accepted October 31, 2013

\begin{abstract}
Wnt signaling has been classified as canonical Wnt/ß-catenin-dependent or non-canonical planar cell polarity (PCP) pathway. Misregulation of either pathway is linked mainly to cancer or neural tube defects (NTDs), respectively. Both pathways seem to antagonize each other, and recent studies have implicated a number of molecular switches that activate one pathway while simultaneously inhibiting the other thereby partially mediating this antagonism. The lipoprotein receptor-related protein Lrp6 is crucial for the activation of the Wnt/ $\beta$-catenin pathway, but its function in Wnt/PCP signaling remains largely unknown. In this study, we investigate the role of Lrp6 as a molecular switch between both Wnt pathways in a novel ENU mouse mutant of Lrp6 (Skax26 ${ }^{\text {1Jus }}$ ) and in human NTDs. We demonstrate that Skax26 ${ }^{\text {m1Jus }}$ represents a hypermorphic allele of Lrp6 with increased Wnt canonical and abolished PCP-induced JNK activities. We also show that $L r p 6^{\text {Skax26-Jus }}$ genetically interacts with a PCP mutant $\left(\right.$ Vang $\left./ 2^{L p}\right)$ where double heterozygotes showed an increased frequency of NTDs and defects in cochlear hair cells' polarity. Importantly, our study also demonstrates the association of rare and novel missense mutations in LRP6 that is an inhibitor rather than an activator of the PCP pathway with human NTDs. We show that three LRP6 mutations in NTDs led to a reduced Wnt canonical activity and enhanced PCP signaling. Our data confirm an inhibitory role of $L$ rp6 in PCP signaling in neurulation and indicate the importance of a tightly regulated and highly dosage-sensitive antagonism between both Wnt pathways in this process.
\end{abstract}

\section{INTRODUCTION}

Wnt signaling plays key roles in regulating various aspects of embryonic development from embryo patterning and cell specification, to regulation of cell movements and tissue polarity (1). Binding of the Wnt ligand to a cell surface receptor can stimulate a range of intracellular signaling pathways, of which the two best studied are the canonical Wnt/ $\beta$-catenin-dependent pathway and the non-canonical planar cell polarity $(\mathrm{PCP})$ pathway $(1,2)$. In the canonical pathway, Wnt binds to Frizzled $(\mathrm{Fz})$ receptor and the lipoprotein receptor-related protein (LRP)-5/6 to induce their clustering on Dishevelled (Dvl) and to form endocytic
'(LRP)-5/6 signalosomes'. These complexes then destabilize the $\beta$-catenin destruction complex that constitutively targets $\beta$-catenin for polyubiquitination and degradation in the proteasome. When the $\beta$-catenin destruction complex is destabilized, $\beta$-catenin accumulates in the nucleus, where it binds to members of TCF/LEF-1 transcription factor family, preventing their repression of gene transcription and functioning as a transcription co-activator $(1,2)$. The $\mathrm{Wnt} / \beta$-catenin pathway has a crucial role in mediating cell proliferation and cell fate determination during development, and defects in this pathway have been strongly associated with many types of cancer in humans (1).

\footnotetext{
${ }^{*}$ To whom correspondence should be addressed at: Department of Obstetrics and Gynecology, CHU Sainte Justine Research Center and University of Montréal, 3175 Cote-Ste-Catherine, Room A711, Montreal, QC, Canada H3T 1C5. Tel: +514 3454931; Fax: +514 3454801; Email: zoha.kibar@, recherche-ste-justine.qc.ca

${ }^{\dagger}$ R.A. and S.L. have contributed equally to the work.
} 
The canonical Wnt $/ \beta$-catenin pathway shares the Fz receptors and the scaffolding proteins (Dvl) with the non-canonical Wnt/ PCP signaling pathway (1). The latter regulates the process of PCP by which cells become polarized in the plane of an epithelium (3). PCP signaling has been well studied in the adult wing hairs and ommatidia (eye units) in Drosophila and includes a group of 'core' PCP genes including $f z$, dsh and vang gogh/strabismus (vang/stbm) (3). Downstream effectors of this pathway include the small GTPases of the RhoA family and JNK that upon activation lead to a variety of cellular responses including cytoskeletal rearrangements (4). In vertebrates, PCP signaling controls the morphogenetic process of convergent extension (CE) during gastrulation and neurulation $(5,6)$. This pathway is highly dosage sensitive where over- or under-expression of PCP genes in zebrafish and Xenopus lead to CE defects $(7,8)$. Studies in animal models and human cohorts have strongly implicated the non-canonical Wnt/PCP signaling pathway in the pathogenesis of neural tube defects (NTDs) (9-11).

NTDs represent a group of very common congenital malformations in humans, affecting 1-2 infants per 1000 births. They are caused by a partial or complete failure of neural tube closure during embryogenesis and can occur at any level along the rostrocaudal axis (9). The most common forms of NTDs are referred to as open NTDs and include anencephaly and myelomeningocele (spina bifida), which result from the failure of fusion in the cranial and spinal region of the neural tube, respectively (9). A number of skin-covered (closed) NTDs are categorized clinically depending on the presence (including lipomyeloschisis, lipomyelomeningocele and meningocele) or absence of a subcutaneous mass (including dermal sinus and caudal agenesis) (12). Population and family studies indicate a complex etiology to NTDs involving environmental and genetic factors. To date, the number, identity and relative contribution of such genes to NTDs remain largely unknown $(9,10)$.

Both Wnt pathways seem to antagonize each other, and inhibiting one will simultaneously activate the other (2). The direction of Wnt signaling and this antagonistic crosstalk are highly dependent on cellular context and on the presence of an intricate and large network of Wnt receptors, co-receptors and regulators (2). Recent studies have identified a group of 'molecular switches' that act on both pathways simultaneously $(2,13-15)$. For example, while Lrp5 or Lrp6 is crucial for the activation of the Wnt/ $\beta$-catenin canonical pathway, it was shown in Xenopus that it could simultaneously inhibit the non-canonical pathway $(16,17)$. Lrp6 is a type I single-span transmembrane protein that belongs to the LDL receptor (LDLR) family, which has diverse roles in metabolism and development (18). It consists of four YWTD $\beta$-propeller domains that are each followed by an EGF-like domain, followed by three LDLR type A repeats, a transmembrane domain and a short intracellular domain (18). Complete inactivation of Lrp6 in mouse generated multiple defects in many organs including eye, limb, heart, neural tube and brain and causes perinatal mortality $(19,20)$. A spontaneous $\operatorname{Lrp} 6^{r s}$ allele caused skeletal malformations, osteoporosis and spina bifida (21) whereas two hypermorphic alleles, one ENU induced $\left(\operatorname{Lrp} 6^{G w}\right)(22)$ and one spontaneous $\left(L r p 6^{C d}\right)$, caused a reduced forebrain size or exencephaly, respectively (22,23). Although the role of Lrp6 in Wnt/ $\beta$-catenin signaling during development is well established, its role in the PCP pathway remains poorly defined.
Here, we conducted in vivo and in vitro studies in mouse and in cell lines to further investigate the role of $\operatorname{Lrp} 6$ as a molecular switch between both Wnt pathways in neural tube closure. We characterized a new ENU hypermorphic allele of Lrp6, Skax $26^{m I J u s}$, that demonstrated an inhibitory role of Lrp6 in PCP signaling as manifested by an increased Wnt canonical activity and abolished PCP-induced JNK activity. We showed that Lrp $6^{\text {Skax26-Jus }}$ genetically interacts with a PCP mutant Vangl ${ }^{L p}$ in PCP signaling in neural tube closure and planar polarity of the inner ear hair cells. Also importantly, our study demonstrates the association of rare and novel mutations in LRP6 with human NTDs. Particularly, we show that three of these mutations caused a reduced Wnt canonical activity and enhanced PCP signaling, thereby confirming a dosage effect of LRP6 in PCP signaling in neurulation.

\section{RESULTS}

\section{Cloning of the gene mutated in Skax26 ${ }^{\text {m1Jus }}$}

Skax26 ${ }^{m I J u s}$ (MGI: 3577510) was identified as a recessive ENU mouse mutant with a homozygous kinky/looped tail phenotype at the Mouse Mutagenesis and Phenotyping Center for Developmental Defects in Texas Medical Center in Houston. This mutant was generated on a mixed C57BL/6J; 129S6/SvEvTac genetic background as part of the chromosome 4 balancer mutagenesis screens (24). G3 mutants from this line were archived as frozen sperm and were consequently recovered at The Jackson Laboratory by using the Assisted In Vitro Fertilization method. A total of two female and five male mice with a normal appearance were generated. To expand the Skax $26^{m l J u s}$ mutant line, these seven founder mice were backcrossed with 129S6/SvEvTac for one generation (backcross 1) followed by four generations of brother-sister mating. A total of 112 N1F1-N1F4 mice were obtained including 11 mice with a kinky/looped tail that were used for subsequent genetic mapping studies.

To map the gene defective in Skax $26^{\text {mIJus }}$, mice with a kinky/ looped tail phenotype were subjected to 3 additional consecutive backcrosses to 129S6/SvEvTac combined with brother-sister matings at each backcross to recover the tail phenotype. A total of 37 mice were genotyped with 149 single nucleotide polymorphisms (SNPs) distributed across the genome and that were informative for both C57BL/6J; 129S6/SvEvTac strains. Homozygosity mapping identified one SNP rs13479079 located at $140.1 \mathrm{Mb}$ at the telomeric end of chromosome 6 that was homozygous for the C57BL/6J allele in $84 \%$ of genotyped mutant mice (Fig. 1A). To fine map this candidate region, we conducted additional genotyping of the same 37 mutant mice with 16 informative SNPs spanning a $23.4-\mathrm{Mb}$ region defined by rs6200835 at $125.7 \mathrm{Mb}$ and rs30113154 at $149.1 \mathrm{Mb}$ at the telomeric end of chromosome 6. Haplotype analysis identified a homozygous region spanning rs3023102 and rs4226339 (haplotypes 1 to 6 ) and segregating with the phenotype in $86 \%$ of genotyped mice (32/37) (Fig. 1B). Importantly, we detected one crossover (haplotype 7) that would place the gene responsible for the Skax26 $6^{\text {IJus }}$ phenotype centromeric to rs3023102 at 135.3 Mb (Fig. 1B). The mutant with this critical crossover along with three other mutant mice (haplotype 8 ) with a kinky/ looped tail phenotype was heterozygous at B6 alleles suggesting 


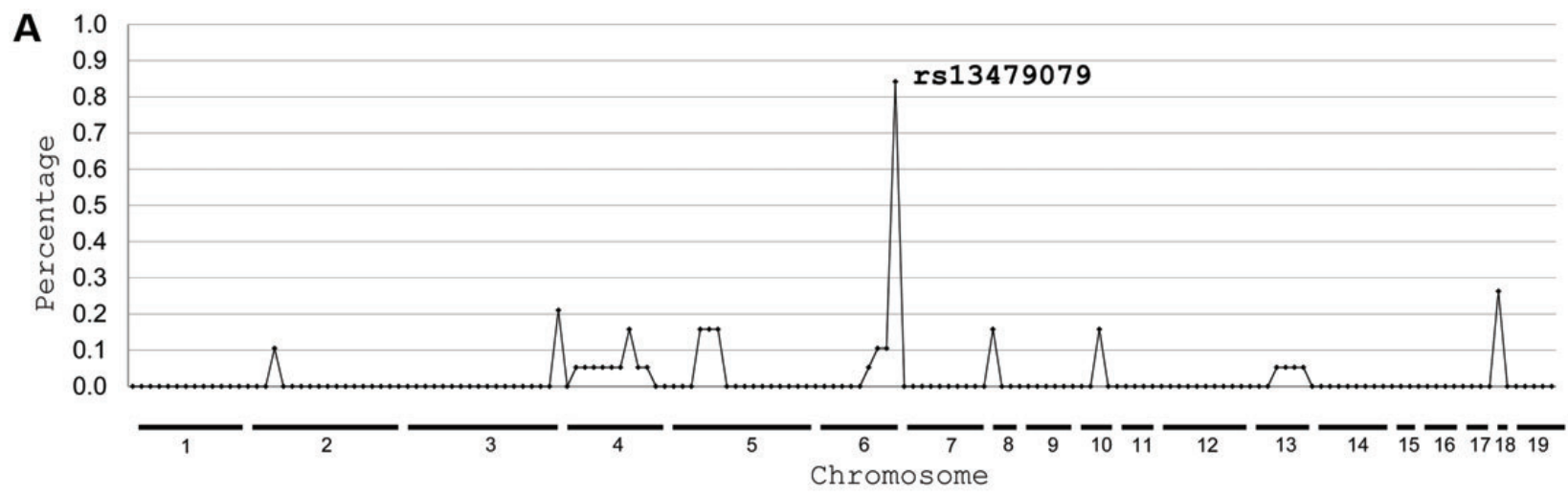

B

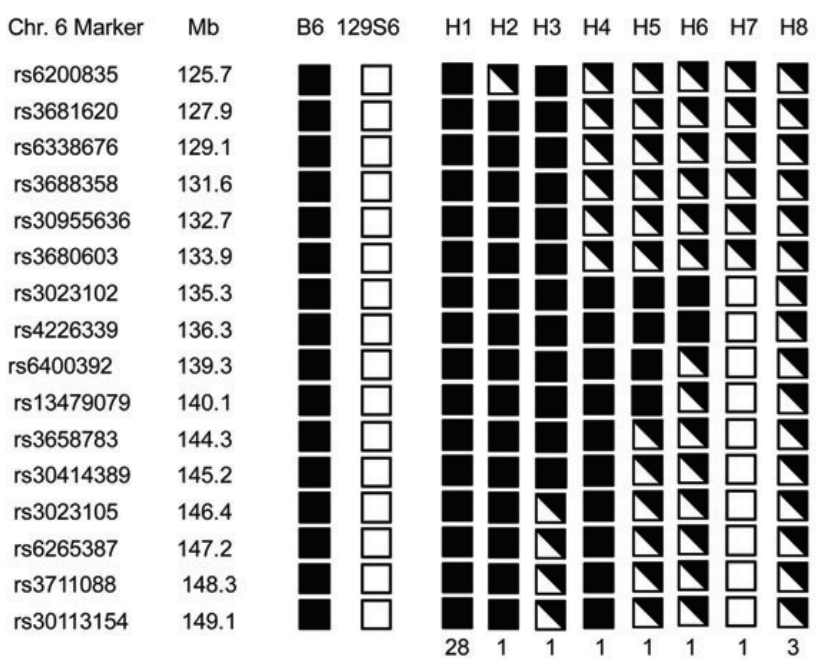

C
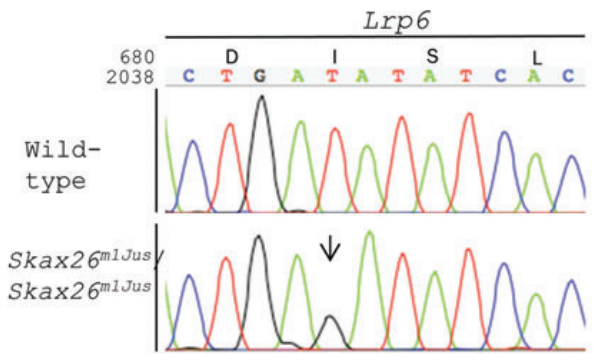

D

hLRP 6

mLrp6

rLrp6

cLrp6

xLrp6

zLrp6

dArrow Ile 681

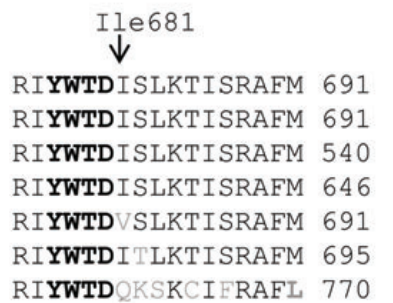

Figure 1. Identification of the gene mutated in $S k a x 26^{m 1 J u s}$. (A) Homozygosity mapping shows linkage of Skax $26^{m 1 J u s}$ to telomeric position of chromosome 6 . The graph shows the percentage of homozygous mice for C57Bl/6J allele for each SNP $(n=149)$ for each chromosome $(n=19)$. (B) Haplotype analysis with 16 SNPs places the Skax26 $6^{m I J u s}$ gene centromeric to rs3023102 at $135.3 \mathrm{Mb}$ on chromosome 6. Black box, C57BL/6J (B6) allele. Open box, 129S6/SvEvTac allele (129S6). Black and open box, heterozygous for B6 and 129S6 alleles. H, haplotype (C) Chromatograms of partial sequences of exon 9 of $\operatorname{Lrp} 6$ show a c.2042T $>\mathrm{G}$ nucleotide change in Skax $26^{\text {mIJus }} /$ Skax $26^{\text {mIJus }}$, which leads to p.Ile681Arg in the encoded protein. (D) Partial protein alignments of mouse Lrp6 with five orthologs. The p.Ile681Arg variant maps to a highly a conserved region following an YWTD repeat (indicated in bold). Accession numbers: Homo sapiens LRP6 (hLRP6), NP_002327.2; Mus musculus Lrp6 (mLrp6), NP_032540.2; Rattus norvegicus Lrp6 (rLrp6), NP_001101362.1; Gallus gallus Lrp6 (cLrp6), XP_417286.3; Danio rerio Lrp6 (zLrp6), NP_001128156.1; Xenopus tropicalis Lrp6 (xLrp6), NP_001079233.1; Drosophila melanogaster Lrp6, (dArrow), NP_524737.2.

that the Skax $26^{m I J u s}$ mutation could cause a phenotype in a heterozygous state at a low penetrance.

Just $0.9 \mathrm{Mb}$ centromeric to rs3023102 resides the Lrp6 gene (accession number: NM_008514.4) that represents an excellent candidate for Skax $26^{m \bar{I} J u s}$. We consequently sequenced the open reading frame and exon-intron junctions of this gene in three Skax $26^{m 1 J u s} /$ Skax $26^{m 1 J u s}$ mutants and three wild-type controls. We identified a homozygous missense mutation c. $2042 \mathrm{~T}>\mathrm{G}$ in exon 9 of Lrp6 in Skax26 $6^{\mathrm{mlJus} / \text { Skax } 26^{\text {mIJus }} \text { that }}$ was absent in parental strains, C57BL/6 and 129S6SvEvTac, and in 30 other inbred strains (Fig. 1C). This mutation leads to p.Ile681Arg that resides in the third $\beta$-propeller domain in the extracellular part of the protein. Ile 681 is highly conserved across vertebrates where it is only replaced by valine in frog Lrp6 and by phenylalanine in fly Arrow (Fig. 1D). While a change to valine or phenylalanine preserves the hydrophobic nature of isoleucine, the change to arginine in Skax $26^{m I J u s}$ is not conservative as it introduces a positive charge and a hydrophilic residue at this otherwise neutral position.
Genotype-phenotype studies were next conducted to assess the penetrance of the Lrp $6^{\text {Skax } 6-J u s}$ p.Ile681Arg mutation. A total of 112 N1F1-N1F4 mice and 99 N5F1 mice were examined for the presence of kinky/looped tail (Table 1). The Lrp $6^{\text {Skax26-Jus }}$ p.Ile681Arg mutation showed a recessive mode of inheritance where all wild-type were phenotypically normal and heterozygous and homozygous adult mice had a looped/kinky tail appearance with a variable penetrance (Table 1). In N1F1-N1F4 mice, the penetrance of the looped/ kinky tail was $3 \%$ in heterozygotes and $11 \%$ in homozygotes. Following four additional backcrosses to129S6SvEvTac, this penetrance increased to $8 \%$ in heterozygotes and $53 \%$ in homozygotes (Table 1). To determine whether the Lrp6 p.Ile681Arg mutation can cause an NTD phenotype, as previously reported for other Lrp6 mutations and knockouts $(19-21,23)$, a total of 30 heterozygous and 47 homozygous E12.5-E18.5 embryos recovered at $\mathrm{N} 5 \mathrm{~F} 1$ were examined macroscopically for the presence of an NTD and none was found to be affected with this malformation (Table 1). 


\section{Functional analysis of Lrp6 ${ }^{\text {p.Ile681Arg in Wnt canonical }}$ and non-canonical pathways}

Lrp6 is a co-receptor of Frizzled that mediates Wnt-induced activation of the transcription factor family TCF/LEF-1 during Wnt canonical $\beta$-catenin signaling (18). We consequently tested the effect of the variant p.Ile681Arg detected in Lrp6 on this signaling pathway using a TCF/LEF-1-responsive Wnt/ $\beta$-catenin reporter assay in mouse embryonic fibroblasts

Table 1. Genotype-phenotype studies in Lrp6 $6^{\text {Skax26-Jus }}$

\begin{tabular}{lccll}
\hline Mice & $N$ & $\begin{array}{l}\text { p.Ile681Arg } \\
\text { genotype at Lrp6 }\end{array}$ & $\begin{array}{l}\text { Phenotype } \\
\text { Kinky/looped } \\
\text { tail }\end{array}$ & $\begin{array}{l}\text { Neural tube } \\
\text { defect }\end{array}$ \\
\hline N1F1-N1F4 adults & 112 & $12+/+$ & 0 & NA \\
& & $30+/-$ & $1(3 \%)$ & NA \\
N5F1 adults & $99-/-$ & $8(11 \%)$ & NA \\
& & $36+/+$ & 0 & NA \\
N5F1 & & $60-/-$ & $3(8 \%)$ & NA \\
E12.5-E18.5 & 91 & $14+/+$ & $32(53 \%)$ & NA \\
& & $30+/-$ & 0 & 0 \\
& & $47-/-$ & $25(63 \%)$ & 0 \\
\end{tabular}

NA, not applicable.
(MEFs) isolated from E13.5 mutant embryos and in mammalian HEK293T cells. Heterozygous and homozygous MEFs showed an increased reporter activity of $\sim 1.4 \times$ and $\sim 3 \times$, respectively, as compared with wild-type fibroblasts $(P<0.05$ and $P<$ 0.00001 , respectively) (Fig. 2A). In HEK293T cells, TCF/ LEF-1 activity was significantly increased to $\sim 2 \times$ in cells transfected with $\operatorname{Lrp} 6^{\text {p.Ile } 681 \mathrm{Arg}} \mathrm{cDNA}$ as compared with wild-type Lrp6 $(P<0.005)$ (Fig. 2D), consistent with results obtained in MEFs. This increased activity of Lrp6 in mutant MEFs or HEK293 T cells was not caused by increased protein expression levels (Fig. 2C and F). These data demonstrate that the Lrp6 ${ }^{\text {p.Ile681Arg }}$ mutant represents a hypermorphic allele that over-activates Wnt canonical $\beta$-catenin signaling.

Lrp6 can act also as an inhibitor of the Wnt non-canonical PCP pathway in Xenopus $(16,17)$. To determine whether the hyperactive $L r p 6^{\text {p.Ile681Arg }}$ allele will also over-inhibit PCP signaling in mouse, we measured the JNK activity in the presence of Wnt5a in wild-type and mutant MEFs using an JNK-AP-1 reporter assay. In wild-type E13.5 MEFs, Wnt5a-induced JNK activity was increased to $2.5 \times$. This activity reduced by half in Lrp $^{\text {Skax26-Jus }}$ heterozygous cells and completely absent in Lrp $^{\text {Skax26-Jus }}$ homozygous cells as compared with wildtype cells $(P<0.05)$ (Fig. 2B). These data confirm that

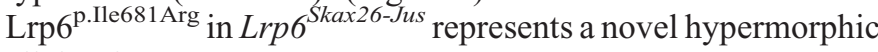
allele of Lrp6.
A

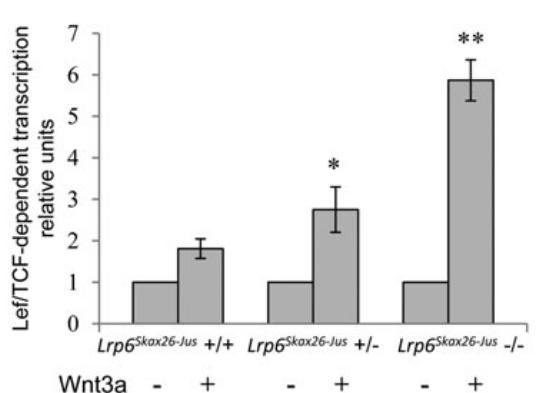

D

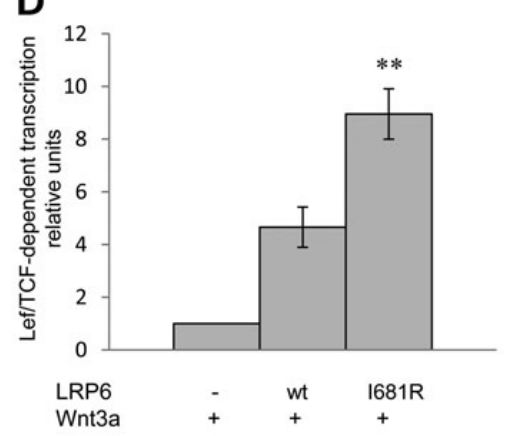

B

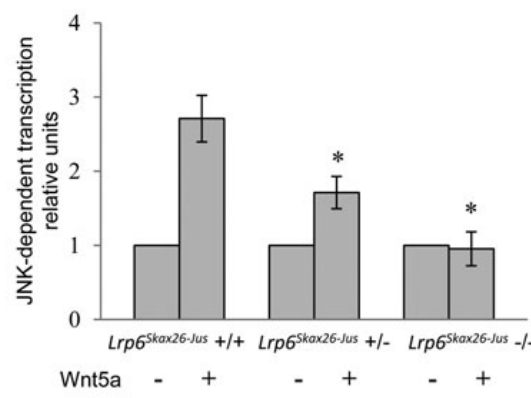

E

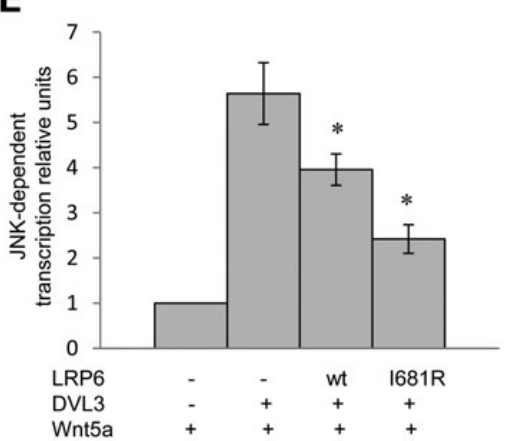

C
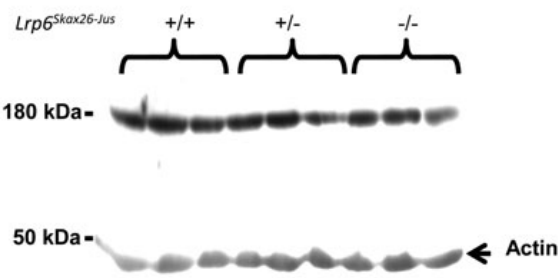

$37 \mathrm{kDa}=$

$\mathbf{F}$

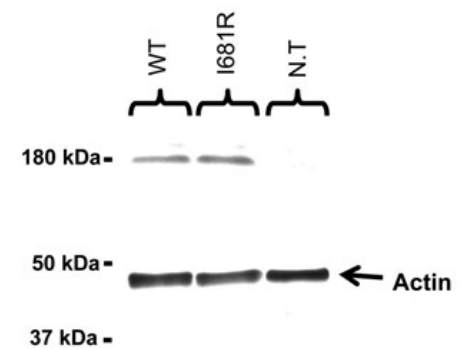

$37 \mathrm{kDa}$ -

Figure 2. Functional validation of the mutant Lrp6 $6^{\text {p.lle681 Arg }}$ in wild-type, heterozygous and homozygous Lrp $6^{\text {Skax } 26-J u s}$ littermates and in HEK293T cells. (A) TCF/ LEF-1 activity was measured in E13.5mouse embryonic fibroblasts (MEFs) in the presence of Wnt3a. Heterozygous $(n=7)$ and homozygous $(n=5)$ MEFs showed a significantly increased reporter activity as compared with wild-type fibroblasts $(n=5)(t$-test, $P<0.05$ and $P<0.00001$, respectively). (B) JNK-AP-1 activity was measured in E13.5 MEFs in the presence of Wnt5a. JNK activity was reduced by half in heterozygous MEFs $(n=7)(P<0.05)$ and completely absent in homozygous cells $(n=4)(P<0.05)$ as compared with wild-type littermates $(n=4)$. $(\mathbf{C})$ Representative western blots of three E13.5 embryos from each genotype $(+/+$,

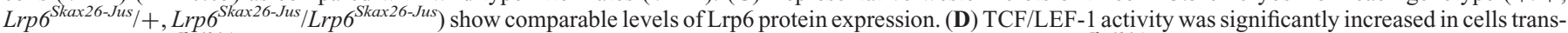
fected with $\operatorname{Lrp} 6^{p .1 l e 681 \mathrm{Arg}}$ cDNA as compared with wild-type $\operatorname{Lrp} 6(P<0.005)$. (E) Co-transfection of the Lrp6 $6^{\text {p.lle681Arg }}$ cDNA with DVL3 resulted in a significant increase in its ability to inhibit Wnt5a-induced JNK activation $(P<0.05)$. (F) Representative western blot of HEK293T cells transfected with $L r p 6^{p . l l e 681 A r g}$ cDNA $^{2}$ shows a protein expression level that was comparable with the wild-type. 
Table 2. Analysis of the neural tube defect phenotype in genetic interaction studies between $\operatorname{Lrp} 6^{\text {Skax26-Jus }}$ and $\operatorname{Vangl2}{ }^{\text {LP }}$

\begin{tabular}{|c|c|c|c|}
\hline Genotype $^{\mathrm{a}}$ & $N$ & $\begin{array}{l}\text { Phenotype } \\
\text { Kinky/looped tail }\end{array}$ & Spina Bifida \\
\hline$+/+;+1+$ & 32 & 0 & 0 \\
\hline$+/+; \operatorname{Vangl} 2^{L p} /+$ & 25 & $10(40 \%)$ & 0 \\
\hline $\operatorname{Lrp}^{\text {Skax26-Jus }} /+;+/+$ & 47 & 0 & 0 \\
\hline Lrp $6^{\text {Skax26-Jus }} /+;$ Vangl2 $2^{L p} /+$ & 45 & $36(80 \%)^{*}$ & $16(36 \%)^{* *}$ \\
\hline
\end{tabular}

${ }^{\mathrm{a}} L r p 6^{\text {Skax26-Jus }}$ and $\operatorname{Vangl} 2^{L p}$ embryos from N1, N4 and N5 were genotyped for the presence of p.Ile681Arg in Lrp6 and p.Ser464Asn in angl $^{L P}$, respectively. ${ }^{*} P<0.005$

${ }^{* *} P<0.001$.

\section{Genetic interaction studies between $\operatorname{Lrp} 6^{\text {Skax26-Jus }}$ and $\operatorname{Vangl} 2^{L P}$}

We next tested for genetic interaction between $\operatorname{Lrp} 6^{\text {Skax26-Jus }}$ and a PCP mutant called $\operatorname{Vang} l 2^{L P}$ that carries a loss of function mutation, p. Ser464Asn, in a core PCP gene called Vangl2 (25). We crossed Lrp $6^{\text {Skax26-Jus }}$ heterozygous or homozygous mice with Vangl2 ${ }^{L p}$ heterozygous mice and examined for the presence of NTDs in double heterozygous embryos. Whereas mice heterozygous for either mutation showed no evidence for NTD, 36\% of the double heterozygotes exhibited spina bifida $(P<0.001)$ (Table 2, Fig. 3A and B). This genetic interaction was specific, as $\operatorname{Vangl} 2^{L P}$ heterozygotes crossed with wild-type littermates

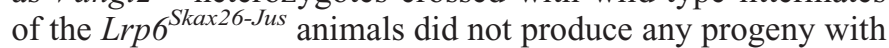
NTDs (Table 2). An increase in frequency of kinky/looped tail was also observed in double heterozygotes (80\%) as compared with single heterozygotes at either loci $(3 \%$ in N1 and $8 \%$ in N5 in Lrp $^{\text {Skax } 26-J u s ~} /+$ and $40 \%$ in $\left.\operatorname{Vangl}^{L P} /+\right)(P<0.005)$ (Tables 1 and 2).

PCP signaling also controls the orientation of hair cells in the cochlea of the inner ear (26). The cochlea has one row of inner hair cells and three rows of outer hair cells, which are interlinked with non-sensory support cells. Each hair cell forms a unique ' $\mathrm{V}$ '-shaped stereociliary bundle and all vertices of these hair bundles point toward the periphery of the cochlea. PCP mutants, including $\operatorname{Vang}^{L} 2^{L P} / \operatorname{Vangl}^{L p}$ homozygotes, display defects in this planar organization $(27,28)$. We next analyzed genetic interaction between Lrp6 $6^{\text {Skax26-Jus }}$ and Vangl2 $2^{L P}$ in inner ear cell polarity. We examined cochleae isolated from wild-type, $\operatorname{Lrp} 6^{\text {Skax26-Jus }} /+, \operatorname{Lrp} 6^{\text {Skax26-Jus }} / \operatorname{Lrp} 6^{\text {Skax26-Jus }}$ and

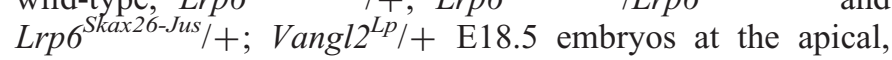
middle and basal regions of the organ of Corti. No statistically significant difference in the orientation of IHC and OHC was detected between wild-type and Lrp $6^{\text {Skax26-Jus }} /+$ (data not shown). In Lrp $6^{\text {Skax26-Jus } / L r p 6^{\text {Skax26-Jus }}}$, bundle orientation was affected as compared with wild-type controls only in the OHC3 layer of the apical region ( $\chi^{2}$ test, $\left.P<0.0001\right)$ (Fig. 3C). In Lrp6 $6^{\text {Skax26-Jus } /+ \text {; Vangl2 }}{ }^{L p} /+$ double heterozygotes, bundle orientation was severely affected in OHC2 and $\mathrm{OHC} 3$ layers at all three regions analyzed except for OHC3 in the middle region of the organ of Corti ( $\chi^{2}$ test, $P<0.01$ for $\mathrm{OHC} 2$ in all regions and $\mathrm{OHC} 3$ at the base and $P<0.0001$ for $\mathrm{OHC} 3$ at the apex) (Fig. 3C). These data confirm a genetic interaction between Lrp6 Skax26-Jus and $\operatorname{Vangl}^{\text {LP }}$ in Wnt noncanonical PCP signaling.

\section{Sequencing analysis of human LRP6 in NTD patients}

The genetic interaction between $\operatorname{Lrp} 6^{\text {Skax26-Jus and } V a n g l 2^{L p}}$ demonstrated in this study as well as the NTD phenotype associated with Lrp6 mouse mutants and the hypothesized role of Lrp6 as an inhibitor of the PCP pathway prompted us to examine LRP6 for a role in the pathogenesis of NTDs in humans. The open reading frame and the exon-intron junction of LRP6 were sequenced in a cohort of 285 Italian patients affected with various types of open and closed forms of NTDs. The exact distribution of NTD type in the patients' cohort is detailed in Materials and methods. We identified four novel rare missense mutations, c. $916 \mathrm{~T}>\mathrm{C}$, c. $1118 \mathrm{~A}>\mathrm{G}$, c. $4157 \mathrm{~T}>\mathrm{G}$ and c. $4622 \mathrm{G}>\mathrm{T}$, in LRP6 in four NTD patients that were not present in 275 ethnically matched controls or in public databases (Supplementary Material, Table S1). Each of these mutations was detected in a heterozygous state and was private. None of the mutations mapped close to the exon-intron junctions and hence most likely do not affect gene splicing. The ORF and exon-intron junctions of LRP6 were also sequenced in 95 Italian controls, and no novel mutation was detected in this gene in controls.

The variant c.916T $>\mathrm{C}$ was detected in a 20 -year-old male affected with a severe form of open NTDs called myelomeningocele in the lumbosacral region of the spine (Supplementary Material, Table S1). This variant changes a highly conserved Tyr residue in the EGF-like domain 1 into histidine (p.Tyr306His) (Fig. 4). A tyrosine-to-histidine change is conservative; however, it could introduce a positively charged amino acid at an otherwise neutral hydrophobic position. The variant c. $1118 \mathrm{~A}>\mathrm{G}$ was detected in a 15-year-old female affected with lumbosacral myelomeningocele and Chiari malformation II (Supplementary Material, Table S1). This variant leads to p.Tyr373Cys in the seventh YWTD $\beta$-propeller domain (Fig. 4A). Tyr373 is highly conserved, and a tyrosine-to-cysteine substitution is not conservative as it introduces a cysteine side chain at this position. The variant c. $4157 \mathrm{~T}>\mathrm{G}$ was detected in a 10-year-old female affected with a severe form of closed NTDs called encephalocele at the parietal lobe of the brain. This type of NTDs is characterized by protrusions of the brain through the skull that are sac-like and covered with membranes (29). The variant c.4157T $>\mathrm{G}$ affects a highly conserved valine residue at position 1386 in the transmembrane domain of the protein (Fig. 4). This leads to a p.Val1386Leu substitution that is conservative as both residues have very similar structures. The variant c. $4622 \mathrm{G}>\mathrm{T}$ was detected in an 11-year-old female affected with a severe form of closed NTDs called caudal agenesis that represents a heterogeneous constellation of anomalies comprising total or partial agenesis of the spinal column, anal imperforation, genital anomalies, bilateral renal dysplasia or aplasia, pulmonary hypoplasia and lower limb abnormalities (12). This variant changes a highly conserved tyrosine at position 1541 in the cytoplasmic domain of LRP6 between the second and third PPPSP phosphorylation motifs and leads to a non-conservative p.Tyr1541Cys substitution.

\section{Functional validation of LRP6 variants detected in NTD patients}

To determine whether any of the four $L R P 6$ variants detected in NTD patients, LRP $6^{\text {p.Tyr306His }}$, LRP $6^{\text {p.Tyr373Cys }}, L R P 6^{\text {p.Val1386Leu }}$ and $L R P 6^{\text {p.Tyr1541Cys }}$, affects the function of LRP6 in both Wnt 
A $+/+$
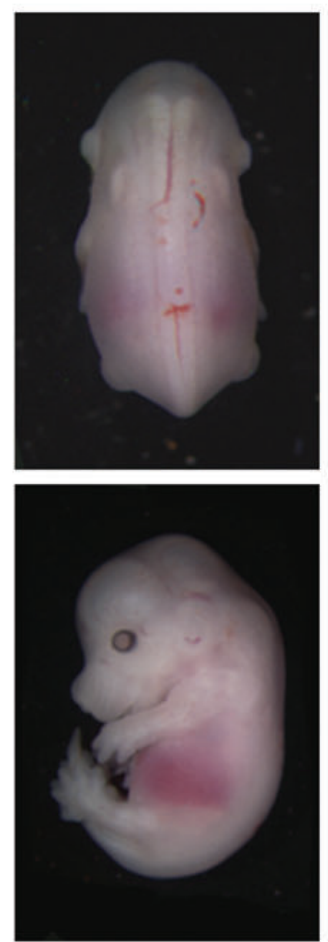

Lrp6 $6^{\text {skax26-Jus/Lrp6 }}$ skax26-Jus
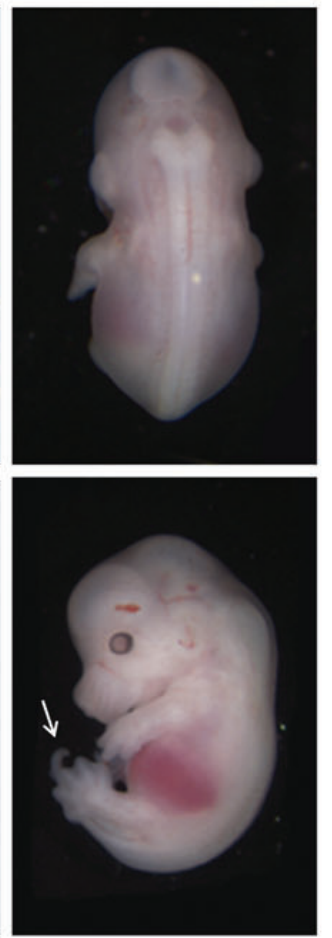

B
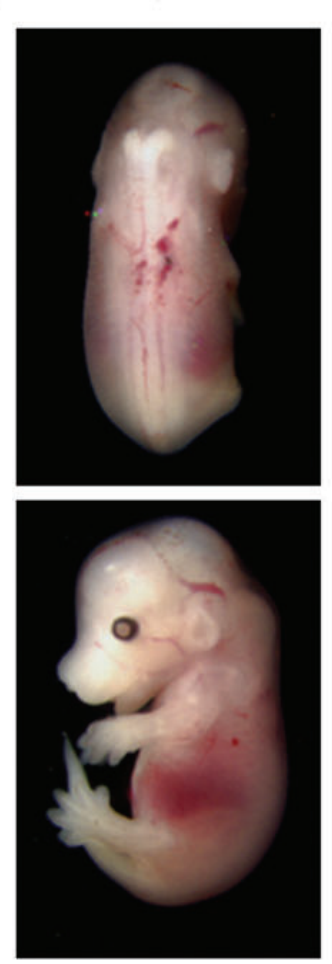

Lrp6 $6^{\text {skax26-Jus/+ ; Vang/2Lo/+ }}$
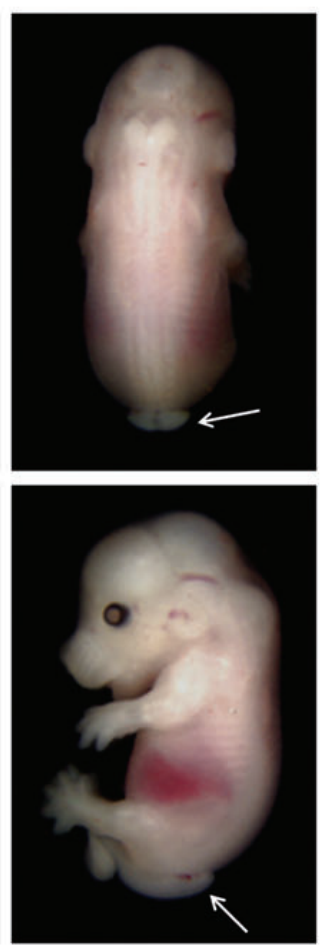

C

$+/+$

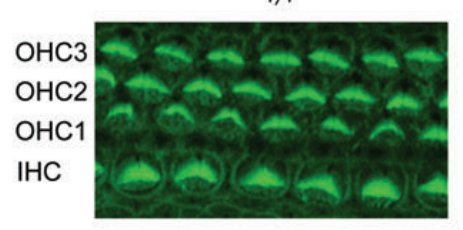

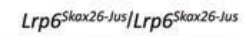

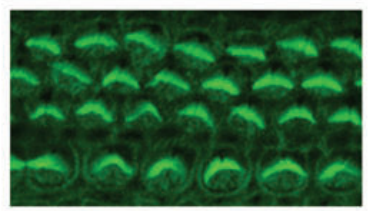

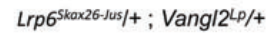
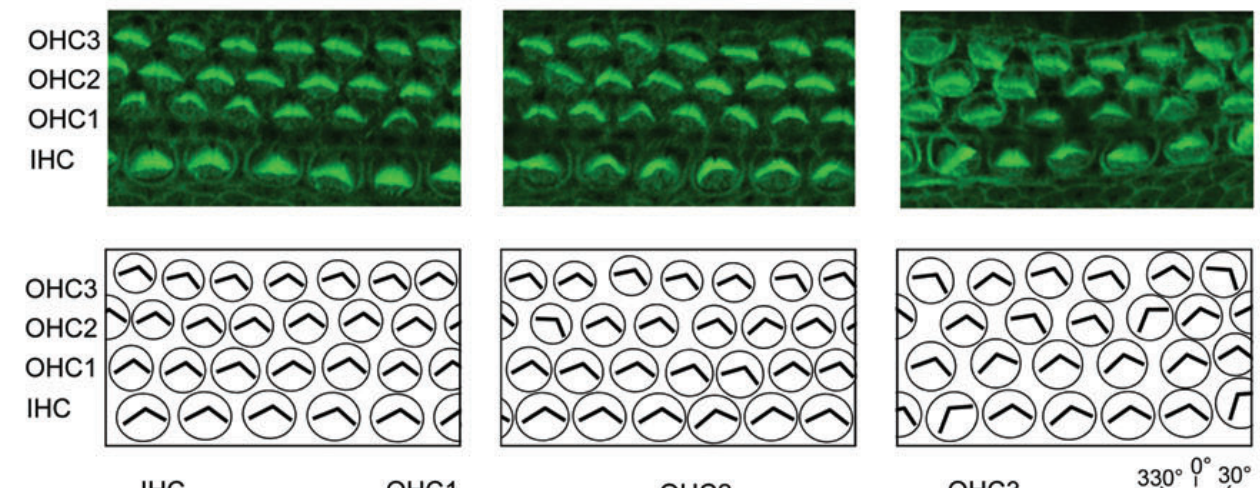

$\mathrm{OHC} 2$

$\mathrm{OHC} 3$

$330^{\circ} 0^{\circ} 30^{\circ}$
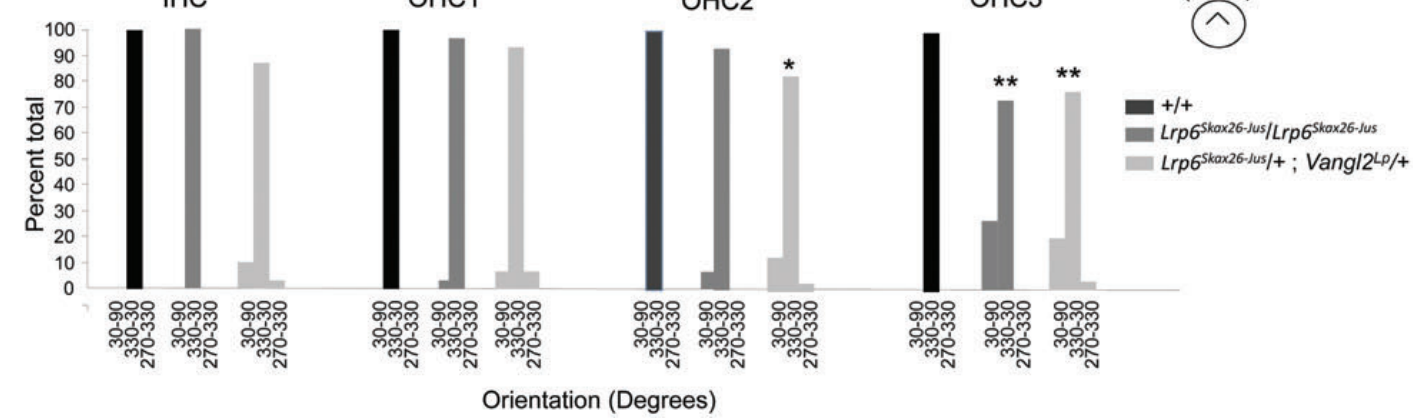

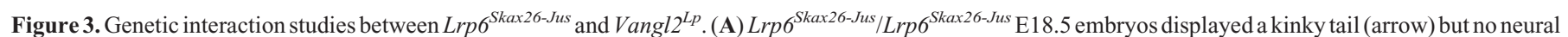

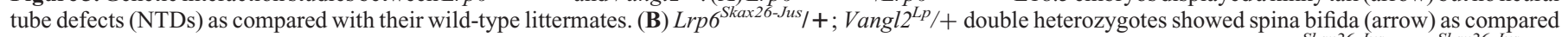

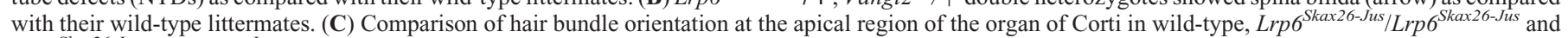

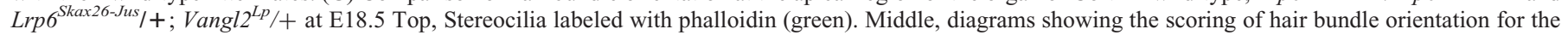

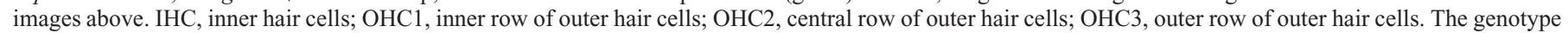
is indicated above each column of panels. Bottom, quantification of the IHC, OHC1, OHC2 and OHC 3 bundle orientations for each of the three genotypes indicated

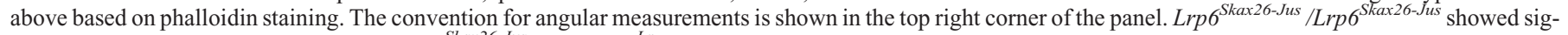

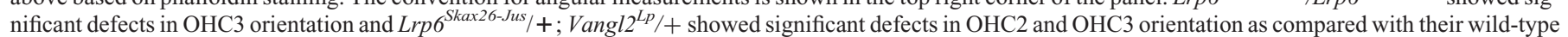
littermates. For statistical analysis, the $\chi^{2}$ test was used to compare the distributions of the various subgroups of hair cells $\left({ }^{*} P<0.01 ;{ }^{* *} P<0.001\right)$. 

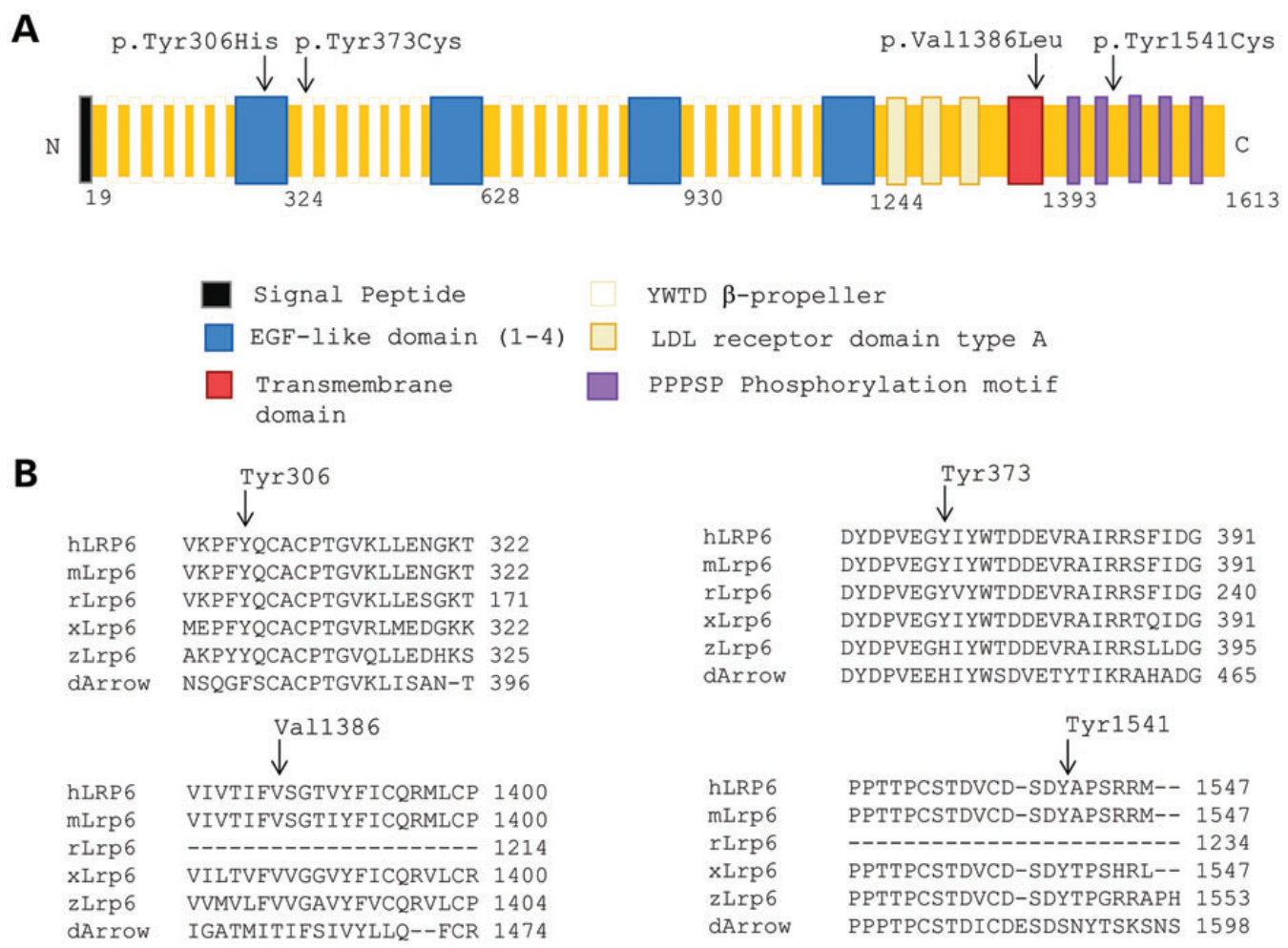

Figure 4. Rare novel mutations in LRP6 in human neural tube defects (NTDs). (A) A schematic diagram of LRP6 showing the approximate locations of the 4 NTD-associated mutations p.Tyr306His, p.Tyr373Cys, p.Val1386Leu and p.Tyr1541Cys. (B) A partial alignment of human LRP6 with five other orthologous sequences. The LRP6 variants found in NTD patients affect conserved residues (indicated by arrows). Accession numbers: Homo sapiens LRP6 (hLRP6), NP_002327.2; Mus musculus Lrp6 (mLrp6), NP_032540.2; Rattus norvegius Lrp6 (rLrp6), NP_001101362.1; Gallus gallus Lrp6 (cLrp6), XP_417286.3; Danio rerio Lrp6 (zLrp6), NP_001128156.1; Xenopus tropicalis Lrp6 (xLrp6), NP_001079233.1; Drosophila melanogaster Lrp6, (dArrow), NP_524737.2.

canonical and non-canonical pathways, we conducted TCF-responsive Wnt/ $\beta$-catenin and AP1-responsive JNK reporter assays, respectively, in mammalian cells (HEK293T). In the presence of Wnt3A, TCF/LEF-1 activity was increased to $\sim 6 \times$ upon transfection of wild-type LRP6, in agreement with previous studies (Fig. 5A). In contrast, TCF/LEF-1 activity was significantly decreased in cells transfected with each of the LRP $6^{\text {p.Tyr } 306 H i s}$, LRP $6^{\text {p.Tyr } 373 \text { Cys }}$ and $L R P 6^{\text {p.Vall } 386 L e u}$ cDNAs, as compared with wild-type $L r p 6$, suggesting a hypomorphic activity for each of these mutants $(P<0.05)$ (Fig. 5A). TCF/LEF-1 activity was not affected in HEK293T cells transfected with the $L R P 6^{\text {p.Tyr } 1541 \text { Cys }}$ (Fig. 5A). Protein expression levels for all LRP6 variants were comparable with the wild-type (Fig. 5B).

While the function of Lrp6 as inhibitor of PCP signaling was deduced from phenotypic effects of abolishing or overexpressing its activity in Xenopus $(16,17)$, how and where exactly it exerts this inhibitory function is not known. We transfected HEK293T with a full-length myc-tagged DVL3 or VANGL2 construct as activators of the PCP pathway in the presence of Wnt5a that was added exogenously. JNK activity was increased to $\sim 5.5 \times$ and $\sim 3.5 \times$ upon transfection of DVL3 and $V A N G L 2$, respectively, in the presence of Wnt5a. This activation was inhibited upon co-transfection of wild-type $L R P 6$ cDNA in a dose-dependent manner $(P<0.05$, at all doses as compared with DVL3 or VANGL2 alone in the presence of Wnt5a) (Fig. 5C). We next tested the effect of the mouse Lrp6 $6^{\text {p.lle681Arg }}$ and the four LRP6 variants detected in human NTDs on their ability to inhibit JNK activation by Wnt5a and
DVL3. Co-transfection of the $\operatorname{Lrp} 6^{\text {p.Ile681Arg }}$ cDNA with DVL3 resulted in a significant increase in its ability to inhibit JNK activation during PCP signaling, confirming its hypermorphic activity $(P<0.05)$ (Fig. 2E). Co-transfection of each of LRP $6^{\text {p.Tyr306His }}$, LRP $6^{\text {p.Tyr373Cys }}$ and LRP $6^{\text {p.Vall } 386 L e u}$ with $D V L 3$ resulted in a significant reduction in its ability to inhibit JNK activation during PCP signaling. On the other hand, LRP $6^{\text {p.Tyr } 1541 \text { Cys }}$ behaved like the wild-type in this assay.

Our results in both readouts assays are consistent with the idea that $\mathrm{LRP}^{\text {p.Tyr306His }}$ LRP $^{\text {p.Tyr373Cys }}$ and LRP6 $6^{\text {p.Val } 1386 \text { Leu }}$ act as hypomorphs and LRP6 ${ }^{\text {p.Tyr1541Cys }}$ acts in Wnt signaling as the wild-type LRP6.

\section{DISCUSSION}

\section{Skax $26^{\text {m1Jus }}$ is a new hypermorphic allele of Lrp6}

Many lines of evidence support our conclusion that Lrp6 is the gene defective in Skax26 $6^{m \text { Jus }}$. Lrp6 maps to the Skax26 $6^{\text {mIJus }}$ candidate region identified by homozygosity mapping on chromosome 6. $\operatorname{Lrp} 6^{\text {Skax26-Jus }}$ carries a disease-specific variant, p.Ile681 Arg, that was absent in parental strains and in 30 other inbred strains. This variant affects Lrp6 function because it caused enhanced Wnt canonical $\beta$-catenin signaling and abolished PCP-induced JNK activation in homozygous MEFs. Ile681 maps to the third YWTD $\beta$-propeller domain, similar to the variant p.Arg886Trp identified in the ENU Lrp6 ${ }^{r s}$ mutant. The $\beta$-propeller-EGF-like domains in Lrp6 appear to bind 


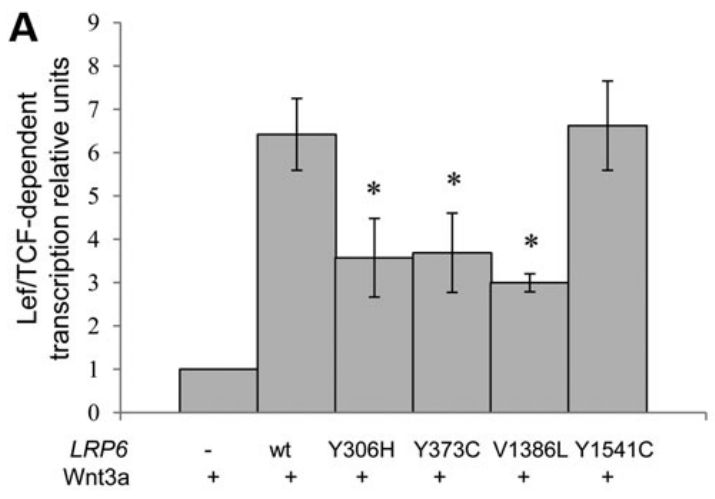

C

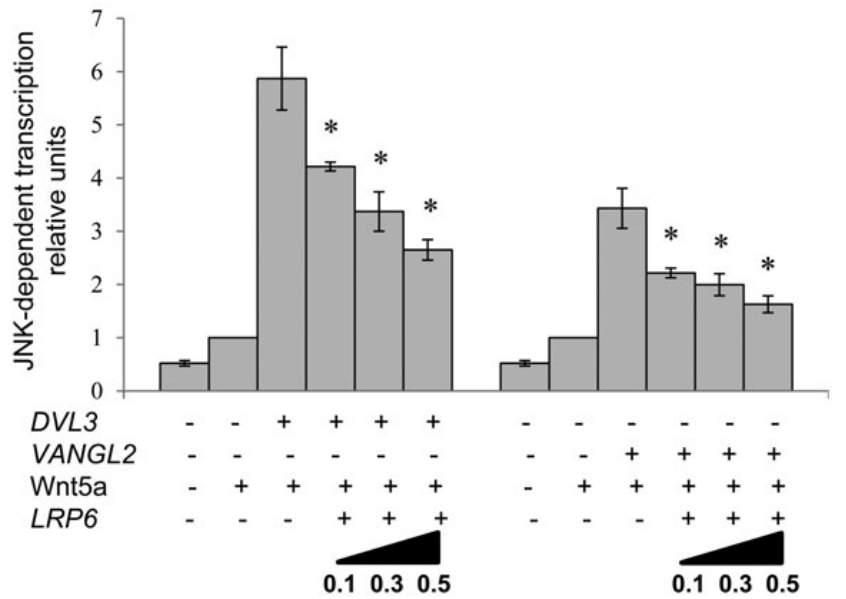

B

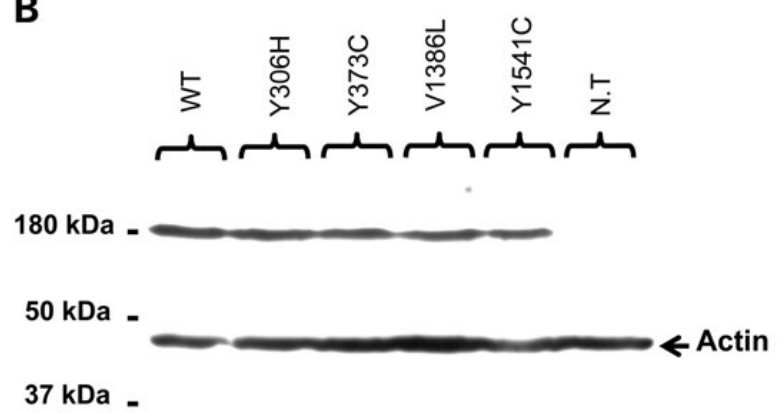

D

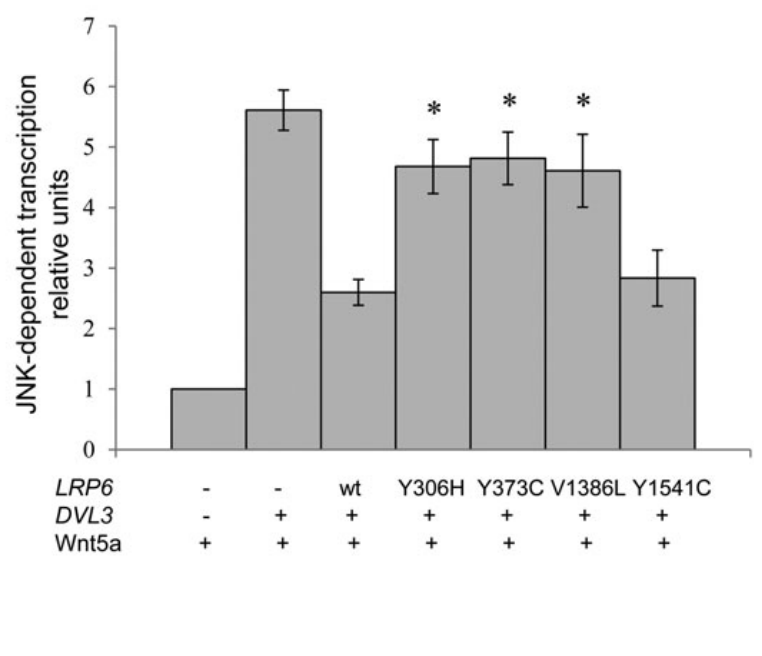

Figure 5. Functional validation of NTD-associated mutations in LRP6 using TCF/LEF-1-responsive Wnt/ $\beta$-catenin and JNK-AP-1 reporter assays. (A) TCF/LEF-1 activity was significantly decreased in cells transfected with each of the $L R P 6^{\text {p.Tyr } 306 \text { His }}, L R P 6^{\text {p.Tyr373Cys }}$ and $L R P 6^{\text {p.Vall386Leu }} \mathrm{cDNAs}$, as compared with wild-type LRP6 $\left(t\right.$-test, $\left.{ }^{*} P<0.05\right)$. This activity was not affected in HEK293T cells transfected with the $L R P 6^{p . T y r 1541 C y s} \mathrm{cDNA}$. (B) A representative western blot analysis of HEK293T cells transfected with each of the four variants shows protein expression levels for all LRP6 variants that were comparable with the wild-type. (C) JNK-AP1-1 activity was significantly inhibited by co-transfection of LRP6 with either DVL3 or VANGL2 in the presence of Wnt5a in a dose-dependent manner $\left(t\right.$-test, $\left.{ }^{*} P<0.05\right)$. (D) Co-transfection of each of LRP6 $6^{\text {.Tyr306His }}$, LRP6 $6^{\text {.Tyr } 373 C y s}$ and LRP6 $6^{\text {.Val1386Leu }}$ cDNAs with DVL3in the presence of Wnt5a resulted in less inhibition of JNK-AP1-1 activity as compared with wild-type $L R P 6$ ( $t$-test, ${ }^{*} P<0.05$ ). LRP $6^{p . T y r 1541 C y s}$ behaved like the wild-type $L R P 6$.

extracellular ligands, and particularly the region encompassing the third and fourth YWTD b-propeller-EGF-like domains of Lrp6 was demonstrated to interact with Dickkopf1 (Dkk1) $(18,30,31)$. Dkk1 antagonizes Wnt/ $\beta$-catenin signaling through interactions with Lrp6 and is able to activate the PCP pathway in both Xenopus and zebrafish (32). The p.Ile681Arg variant in Lrp6 could affect its interaction with Dkk1 or other unknown mediators leading to over-activation of the Wnt pathway and inhibition of the PCP pathway. Additional biochemical and in silico tests are needed to test the potential pathogenic effect of this variant.

All previously studied mouse mutants at $\operatorname{Lrp} 6$ except $\operatorname{Lrp} 6^{G w}$ were reported to suffer from severe developmental anomalies in various organs including eye, limb, heart, neural tube and brain (19-21,23). We did not encounter any severe malformation in either heterozygous or homozygous $\operatorname{Lrp} 6^{\text {Skax26-Jus embryos, sug- }}$ gesting that either this allele could exert a milder effect on protein function or this could reflect the presence of genetic modifiers in the various backgrounds of the Lrp6 mutants. While Lrp $6^{\text {Skax26-Jus }}$ is on a mixed C57BL/6J; 129S6/SvEvTac background, the other $\operatorname{Lrp} 6$ alleles, $\operatorname{Lrp} 6^{c d}, \operatorname{Lrp}_{6}^{G w}$ and $\operatorname{Lrp} 6^{r s}$, were on mixed DBA/2;A/J (23), $\mathrm{C} 3 \mathrm{H} / \mathrm{HeH}$ (22) and mixed BALB/C; C57BL/6J (21) backgrounds, respectively.

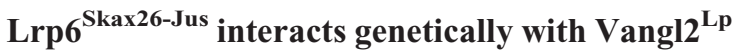

The genetic interaction between $\operatorname{Lrp} 6^{\text {Skax26-Jus }}$ and $\operatorname{Vangl2^{Lp}}$ in neural tube closure and inner ear hair cells' planar polarity provides evidence that both genes function in a common genetic pathway or in two parallel pathways to regulate these developmental processes. Spina bifida was absent from single heterozygotes and was detected in $36 \%$ of their double heterozygous littermates. These data show that the mutant kinky/looped tail and NTD phenotypes in Vangl2 $2^{L p}$ caused by diminished PCP signaling were enhanced by a hyperactive $\operatorname{Lrp} 6^{\text {Skax26-Jus }}$ allele that over-inhibits this pathway. These results also strongly suggest that the tail kink or looping observed in Lrp6 $6^{\text {Skax26-Jus }}$ heterozygotes is associated with a gain of Lrp6 function as an inhibitor of the Wnt non-canonical PCP pathway because of the Lrp6 ${ }^{\text {p.Ile681Arg }}$ mutation, rather than an effect of an increased activation of the Wnt/ $\beta$-catenin canonical pathway. Our 
observations are supported by a recent study in gain $\left(\operatorname{Lrp} 6^{c d}\right)$ and loss ( Lrp6-/-) of function mouse lines that demonstrated Lrp6 can mediate non-canonical pathways involving RhoAdependent mechanisms during neurulation (33).

Genetic interaction between $\operatorname{Lrp} 6^{\text {Skax26-Jus }}$ and $\operatorname{Vangl}^{L p}$ does not seem as strong as that reported between $\operatorname{Vangl}^{L P}$ and mouse mutants at other PCP loci notably Scribblel (Circle or Crc) and Dvl3. A subset of Vangl2: Scribble1 and Vangl2:Dvl3 double heterozygotes developed the more severe craniorachischisis where the neural tube remains open throughout the spinal cord $(34,35)$. On the other hand, Vangl2:Ptk7 (another PCP gene) double heterozygotes develop spina bifida (36), suggesting that either Lrp6 or PTK7 alleles exert a weaker effect on protein function or the dosage of Lrp6 or Ptk7 is less limiting than that of Scribl or $\mathrm{Dvl3}$ or that there may be different genetic modifiers in the various background strains.

\section{Novel rare mutations in $L R P 6$ are associated with human NTDs}

We and others have previously demonstrated an important role for PCP signaling in the pathogenesis of NTDs where novel and rare mutations in PCP genes including VANGL1, PK1, FZD6, FUZZY, SCRIBBLE1 and CELSRI were associated with NTDs in a subset of patients (37-42). All these genes are activators of the PCP pathway, and some mutations were hypothesized to be hypomorphic. In this study and for the first time, we demonstrate the presence of rare and novel mutations associated with NTDs (1.4\% or 4 in 285 patients) in an inhibitor of the PCP pathway that is LRP6. This frequency of $1.4 \%$ of novel potentially pathogenic mutations in $L R P 6$ in NTD patients is very similar to the previously reported mutation frequencies for other PCP genes in NTDs. All four patients were affected with severe forms of open (2 MMC) and closed (1 encephalocele, 1 caudal agenesis) NTDs. As compared with other PCP genes previously associated with NTDs, no LRP6 mutation was detected in the mild forms of closed spinal NTDs. Re-sequencing analysis of LRP6 in a larger cohort is needed to further investigate this observation.

Our results in both Wnt canonical and non-canonical readouts assays are consistent with the hypothesis that LRP6 $6^{\text {p.Tyr306His }}$, LRP6 $^{\text {p.Tyr373Cys }}$ and LRP6 $6^{\text {p.Val1386Leu }}$ act as hypomorphs and LRP6 $^{\text {p.Tyr1541Cys }}$ acts in Wnt signaling as the wild-type LRP6. The latter is either not pathogenic or it exerts a very mild effect that could not be detected by these reporter assays. Enhanced or diminished PCP signaling was previously demonstrated to cause PCP defects but only in zebrafish and frog models. Our study indicates the importance of a precise regulation of dosage in PCP signaling in neural tube closure in humans.

The molecular impact of these three hypomorphic variants on the function of LRP 6 can be deduced from the known function of the specific domains of the protein to which they map. Lrp6 $6^{\text {P.Tyr306His }}$ and Lrp6 ${ }^{\text {p.Tyr373Cys }}$ are located in the first EGF-like domain and in the second propeller domain of LRP6, respectively. The first two YWTD $\beta$-propeller-EGF-like domains bind to canonical Wnts and seem to mediate the Wnt-Fz interaction during Wnt canonical signaling (18). These two domains also bind to the Wise family of proteins, which antagonize $\mathrm{Wnt} / \beta$-catenin signaling through interactions with Lrp6 (31). LRP6 $6^{\text {p.Tyr306His }}$ and LRP6 $6^{\text {p.Tyr373Cys }}$ might alter the interaction of LRP6 with Wnt-Fz negatively or with Wise proteins positively to reduce the $\mathrm{Wnt} / \beta$-catenin signaling activity and to reciprocally activate the non-canonical PCP pathway. The variant LRP $^{\text {p.Val1386Leu }}$ maps to the transmembrane domain of LRP6. Leucine is a hydrophobic amino acid possessing similar structure to valine but longer side chain. This mutation was predicted to be possibly damaging with Polyphen despite the fact that it is a substitution to a similar amino acid. Clearly, additional tests are needed to investigate and better define the pathogenic nature of these four LRP6 variants.

\section{Canonical Wnt/及-catenin and non-canonical Wnt/PCP signaling: two antagonistic and highly dosage-sensitive pathways}

Genetic studies in various animal models demonstrated the existence of a reciprocal antagonism between both Wnt pathways where inhibiting one pathway will simultaneously activate the other one. For example, in zebrafish, maternal loss of wnt $5 b$ resulted in ectopic $\beta$-catenin signaling and a consequent increase in dorsal cell fates $(43,44)$. In Xenopus, knockdown of Lrp6 and its homolog $\operatorname{Lrp} 5$ resulted in PCP defects that were rescued by knockdown of non-canonical XWnt5a and XWnt11 $(16,17)$. In mouse, knockdown of Lrp6 led to PCP defects including exencephaly and heart defects, which were rescued by loss of $W n t 5 a$, suggesting that these phenotypes could result from noncanonical Wnt gain-of-function (45). In this study, we identified four mutations in Lrp6 orthologs (one in mouse and three in humans) in NTDs that affected both Wnt pathways in a reciprocal manner, thereby presenting additional evidence for the existence of such dosage-sensitive antagonism between Wnt pathways in neurulation.

\section{LRP5 and LRP6: homologs with a pleiotropic role in human diseases}

Mutations in LRP6 gene have been implicated in late-onset Alzheimer's disease and familial coronary artery disease associated with diabetes and osteoporosis syndrome $(46,47)$. In this study, we extend the mutational spectrum of LRP6 to another complex trait, NTDs, demonstrating a crucial but pleiotropic role of this gene in developmental diseases. LRP6 has a homologous gene $L R P 5$ that shares 73 and $64 \%$ identity in extracellular and intracellular domains, respectively (18). Lrp5 knockout mice have normal embryogenesis and grow to adulthood but show osteoporosis and some metabolic abnormalities. An allelic series of mouse mutants suggested that Lrp5 and Lrp6 share significant overlapping functions but that Lrp6 plays a more crucial role, at least during embryogenesis (18). In humans, LRP5 mutations underlie familial osteoporosis, high bone density syndromes and ocular disorders (18). Interestingly, Lrp 5 was also shown to act as a physiologically relevant inhibitor of non-canonical Wnt PCP signaling during Xenopus development in vivo (16). These studies represent $L R P 5$ as a good candidate gene for human NTDs. Additional molecular genetic studies of LRP5 and LRP6 in human NTD cohorts and animal models are needed to better dissect their roles in normal and abnormal neurulation. 


\section{MATERIALS AND METHODS}

\section{Maintenance and phenotyping of mice}

The Skax2 $6^{\text {m1Jus }}$ mouse mutant (MGI:3577510) with a looptail phenotype was generated and identified as part of the chromosome 4 balancer mutagenesis screens at the Mouse Mutagenesis and Phenotyping Center for Developmental Defects in Texas Medical Center in Houston (http://www.mouse-genome.bcm. tmc.edu). The study design of the chromosome 4 balancer chromosomes and the mutagenesis screens have been described elsewhere in details (24). Briefly, mice homozygous for the chromosome 4 balancer chromosome (on a C57BL/ $6 \mathrm{BrdTyr}-/-\mathrm{B} 6$-albino genetic background), which could be visually recognized by their dark brown coat because of the presence of tyrosinase and K14-agouti transgenes at the inversion breakpoints, were crossed with ENU-injected C57BL/ $6 \mathrm{BrdTyr}-/-$ (albino) mice. G1 pups that were heterozygous for a potential mutation and the balancer (light brown coat) were backcrossed with balancer homozygotes (dark brown coat). G2 mice that were heterozygous for the inversion (light brown coat) were intercrossed, and the offspring (G3) were analyzed. Skax $26^{\text {mIJus }}$ was identified as a recessive mouse mutant with a looped tail phenotype in the G3 cross that failed to segregate to chromosome 4. Skax $26^{\text {m1Jus }} \mathrm{G} 3$ mutants were archived as frozen sperm and were consequently recovered at The Jackson Laboratory (http://jaxmice.jax.org/services/) by using the Assisted In Vitro Fertilization (AIVF) method with C57BL/6J oocytes. The Skax $26^{\text {m1Jus }}$ mutant stock was generated and maintained by brother-sister matings. For genetic mapping studies, Skax $26^{\text {mIJus }}$ mutants with a kinked/looped tail phenotype were backcrossed with129S6/SvEv for four consecutive times, and at each outcross, the 'looped' or 'kinked' tail recessive phenotype was recovered by brother-sister intercrosses.

Two $\operatorname{Vangl} 2^{L p} /+$ males on a mixed A/J:B6 background were obtained from Philippe Gros (McGill University, Montreal, Quebec) and were maintained by crosses to C57BL/6J mice.

Mice were examined macroscopically for the presence of a severely 'kinked' or 'looped' tail. Embryos were recovered at various stages and examined for the presence of NTDs.

The protocol relevant to support this study was approved by the Institutional Committee for Animal Care in Research of the Research Center of Sainte Justine Hospital.

\section{DNA extraction and genotyping}

Genomic DNA was extracted from mouse tail or embryonic yolk sac using the EZ-10 Spin Column Animal DNA Mini-Preps kit (BS628, Bio Basic Inc. Canada, ON). Phenotype and genotype were confirmed for all mice included in the study. For the Skax $26^{\text {mIJus }}$ mice, genotyping was done by PCR amplification using genomic DNA. Exon 9 of Lrp6 (471 bp) was amplified using intronic primers $5^{\prime}$ catgaaacaaatatgtcetgett $3^{\prime}$ and $5^{\prime}$ tcagtgtgacttcccaccagc $3^{\prime}$. Direct dye terminator sequencing of PCR products was done using Applied Biosystem's 3730xl DNA Analyzer technology. The p.Ile681Arg mutation was also detected by EcoRV digestion on Lrp6-exon9 PCR product and visualized on $2 \%$ agarose gel. For the $\operatorname{Vang}^{L} 2^{L P}$ mutation, genotyping was done by PCR amplification on genomic DNA for Vangl2-exon 7 (360 bp) as previously described (48).

\section{Homozygosity mapping}

For genetic mapping studies, a total of 180 SNPs distributed across the genome were selected for genotyping 37 mice (11 N1F1-N1F4, 5N2F1, 2 N3F1 and 19N3F1). These markers were informative for both C57BL/6J; 129S6/SvEvTac strains and average intermarker distance was $13 \mathrm{Mb}$. Of these, 31 markers failed to genotype. To fine map the Skax $26^{\text {m1Jus }}$ candidate region, additional genotyping of the same 37 mutant mice was done with 19 informative SNPs spanning a 23.4-Mb region defined by rs6200835 and rs30113154 at the telomeric end of chromosome 6 . The average intermarker distance was $1.5 \mathrm{Mb}$. Of these, three failed to genotype. Genotyping was done using the Sequenom iPlex Gold technology at the McGill and Genome Quebec Innovation Center.

\section{Patients and controls}

The patients' cohort consisted of 285 Italian patients recruited at the Spina Bifida Center of the Gaslini Hospital in Genoa, Italy. All patients were affected with non-syndromic or isolated NTDs. The distribution of the major NTD forms in this cohort was as follows: 6 cranial closed NTDs (6 encephalocele), 153 open spinal NTDs (153 myelomeningocele) and 126 closed spinal NTDs (17 Lipomyeloschisis, 18 Lipomyelomeningocele, 38 caudal agenesis, 28 lipoma, 7 tight filum terminale, 2 myelocystocele, 1 meningocele, 11 diastematomyelia, 1 meningocele and 3 lipomyelocele). Myelomeningocele was the major NTD present in this cohort at a frequency of $44 \%$. All NTD patients included in this cohort were white Caucasians. The control group consisted of 275 Italian healthy individuals with no NTD family history. All research involving human participants was approved by the Local Ethics Committee of the Institute of Gaslini, Genoa, Italy. Written informed consent was obtained from all participating individuals.

\section{Sequencing analyses}

The coding exons of mouse Lrp6 (accession number: NM_008514.4) and LRP6 (accession number: NM_002336.2) were amplified from genomic DNA by PCR using primers flanking the exon-intron junctions. Primers' sequences and PCR conditions are available upon request. Direct dye terminator sequencing of PCR products was carried out using the ABI Prism Big Dye Systems at the McGill and Genome Quebec Innovation Center. Samples were run on ABI 3700 automated sequencer and analyzed using the SeqMan ${ }^{\circledR}$ sequence assembly and SNP discovery software (from DNASTAR ${ }^{\circledR}$ ).

Human LRP6 mutations were verified for absence in 275 ethnically matched controls by sequencing analysis and in 3 public databases dbSNP (http://www.ncbi.nlm.nih.gov/snp), the 1000 genome project (http://www.1000genomes.org) and the NHLBI GO Exome Sequencing Project (http://snp.gs.wa shington.edu/EVS/). Novel rare mutations $(<1 \%)$ were confirmed in a second round of sequencing of the original DNA sample. For novel missense mutations absent in controls and in public databases, their effect on the protein was predicted in silico using the PolyPhen (Polymorphism Phenotyping; http ://genetics.bwh.harvard.edu/pph/) and SIFT (Sorting Intolerant 
From Tolerant; http://sift.jcvi.org/) programs. Multiple alignments of orthologous Lrp6 proteins were done using ClustalW.

\section{Cochlear immunohistochemistry and analysis of stereociliary bundle orientation}

Cochlea from E18.5 embryos were isolated under microscope and flat mounted, then fixed in $4 \%$ paraformaldehyde for $1 \mathrm{~h}$ at room temperature and washed $3 \times$ in phosphate-buffered saline (PBS). Immunohistochemical staining was done as previously described. At least 30 cells for each row at the apical, middle or basal region of the organ of Corti were used for quantification per sample. To determine stereociliary bundle orientation, a line was drawn through the middle of the ' $\mathrm{V}$ '-shaped stereocilia (bisecting line). The angle formed between this line and the line parallel to the mediolateral axis was used for quantifications. In wild-type animals, this angle is close to 0 degrees. Stereocilia with a deviation from normal of $>30^{\circ}$ were considered to be misoriented. For statistical analysis, the $\chi^{2}$ test was used to compare the distributions of the various subgroups of hair cells.

\section{Preparation of cell lysates and immunoblot analysis}

After $48 \mathrm{~h}$ in culture, cells were rinsed in ice-cold PBS and collected in cold lysis RIPA buffer $(50 \mathrm{~mm}$ Tris $-\mathrm{HCl} \mathrm{pH} 7.4$, $150 \mathrm{~mm} \mathrm{NaCl}, 1 \%$ Triton X-100, 1\% Sodium deoxycholate, $0.1 \%$ SDS, 1 mm EDTA) supplemented with protease inhibitors and spun at $13000 \mathrm{~g}$ at $4 \mathrm{C}$ for $15 \mathrm{~min}$. Protein concentrations of the supernatant were determined using the bicinchoninic acid assay. Endogenous Lrp6 in MEFS was detected using phospho-specific LRP6 antibodies to the A site (S1490) obtained from Cell Signaling Technology (catalog number 2568) and the VSVG-tagged hLRP6 in HEK293T cells using polyclonal anti-VSVG antibody obtained from Sigma-Aldrich Corporation ( catalog number V4888). $\beta$-actin antibody was from NOVUS Biologicals, LLC ( catalog number NB600-501); secondary antibody to mouse (catalog number ab6789) and to rabbit (catalog number ab6721) were obtained from abcam.

\section{Cell cultures and cDNA expression constructs}

All cells were cultured in Dulbecco's Modified Eagle's medium with $10 \% \mathrm{FBS}$ and $1 \%$ antibiotic and antimycotic cocktail. The $\mathrm{L}$ cells that produce Wnt3a were a generous gift from Dr. Sylvain Chemtob and those producing Wnt5a from Nicolas Pilon. To collect the conditioned medium (CM) (Wnt3a or Wnt5a) from cultures of Wnt3a/5a-producing L cells, we seeded these cells at a density of $1 \times 10^{6}$ cells in a 94-mm dish containing a 1:1 mixture of DMEM and HAM F12 supplemented with $10 \%$ FCS and cultured them for 4 days. Then, CM was harvested, centrifuged at $1000 \mathrm{~g}$ for $10 \mathrm{~min}$, and filtered through a nitrocellulose membrane.

The pTOPFLASH (pGL3) vector was a generous gift from Nicolas Pilon. The pCS2-VSVG-tagged wild-type LRP6 vector was kindly provided by Dr. Xi He. Mouse Lrp6, human $D V L 3$ and human VANGL2 constructs were subcloned into the pCDNA3.1 Myc/His A and pCS2 + expression vectors, respectively, the SLIC method (49). Site-directed mutagenesis was performed using the PfuTurbo ${ }^{\circledR}$ DNA Polymerase (Stratagene) as directed. Primers used for cloning and for site directed mutagenesis are provided upon request.
TCF/LEF-1-responsive Wnt/B-catenin- and JNK-AP-1-dependent reporter assays

All mammalian cell transfections in HEK293T cells were done using either Lipofectamine ${ }^{\circledR} 2000$ Transfection Reagent or Polyethylenimine and were performed in triplicates. For the Lef/Tcf reporter assay, HEK293T cells were transfected with [50 ng TOPFLASH /pGL3-OT, $10 \mathrm{ng}$ pRL-TK and $100 \mathrm{ng}$ of wild-type or mutant $L R P 6$ constructs or pCS2 vector alone as control] per well in 24-well plates. Following transfection, cells were grown in DMEM $/ 10 \% \mathrm{FBS}$ overnight and in Wnt3a-enriched medium for another overnight before measuring the TCF/LEF-1 activity.

For the JNK-AP-1 reporter assay, HEK293T cells were transfected with a total of [50 ng TOPFLASH /pAP1-Luc, $100 \mathrm{ng}$ DVL3 or $500 \mathrm{ng}$ VANGL2, $10 \mathrm{ng}$ pRL-TK and increasing amounts (100-500 ng) of LRP6 constructs or pCS2 empty vector control] per well in 24 -well plates. For functional validation of LRP6 mutations, HEK293T cells were transfected with [100 ng pAP1-Luc, $10 \mathrm{ng}$ TK-Renilla and $100 \mathrm{ng}$ of DVL3 constructs alone or with $500 \mathrm{ng}$ LRP6 wild-type or empty pCS2 vector alone as control] DNA per well in 24-well plates. The assay was performed using the PathDetect Kit (Stratagene) according to manufacturer's instructions (catalog number 219074-51).

MEFs were prepared from N5F1 embryos at E13.5 using standard procedures and were transfected with $10 \mathrm{mg}$ reporter vector (pGL3-OT or pAP1-Luc) and $5 \mathrm{mg}$ pRL-TK/60-mm dish by electroporation and incubated overnight in DMEM/ $10 \% \mathrm{FBS}$ and another overnight in Wnt5a- or Wnt3a-conditioned medium.

pRL-TK (Promega, catalog number E224A), which encodes a Renilla luciferase gene downstream of a minimal HSV-TK promoter, was included in each transfection to control for transfection efficiency. Dual luciferase reporter assays were performed as described by the manufacturer (Promega, catalog number E1960).

\section{Statistical analysis}

Two-tailed Fisher's exact test was used for statistical analysis of genetic interaction data between $\operatorname{Lrp} 6^{\text {Skax 26-Jus }}$ and $\operatorname{Vangl}^{L p}$. All reporter activity experiments were carried out at least three times. Data were represented as mean \pm SE. Significance of differences between two groups was tested either by $\chi^{2}$ test or Student's $t$-test. A $P$ value of $<0.05$ was considered as significant.

\section{SUPPLEMENTARY MATERIAL}

Supplementary Material is available at $H M G$ online.

\section{ACKNOWLEDGEMENTS}

We thank all participants in this study. We also thank Corinne Saint Denis, Genevieve Lefebvre, Ruiu Ilaria and Mara Uglietta for technical assistance. We thank Drs. Philippe Gros (McGill University, Montreal, Quebec) for providing $\operatorname{Vangl}^{L p}$ mice, Nicolas Pilon (University of Québec in Montréal) for providing L Wnt5a cells and the pTOPFLASH (pGL3) vector, Sylvain Chemtob (University of Montréal) for providing L Wnt3a cells and $\mathrm{Xi} \mathrm{He}$ (Harvard Medical School) for providing the 
pCS2-VSVG-tagged wild-type LRP6 vector. We also thank ASBI (Associazione Spina Bifida Italia).

\section{Conflict of Interest statement. None declared.}

\section{FUNDING}

Z.K. has a salary award from the 'Fonds de Recherche du Québec - Santé' and is funded by the Canadian Institutes for health Research. M.J.J. is funded by NIH grants U54 HD39372 and R01 CA115503.

\section{REFERENCES}

1. Niehrs, C. (2012) The complex world of WNT receptor signalling. Nat. Rev. Mol. Cell Biol., 13, 767-779.

2. Chien, A.J., Conrad, W.H. and Moon, R.T. (2009) A Wnt survival guide: from flies to human disease. J. Invest. Dermatol., 129, 1614-1627.

3. Maung, S.M.T.W. and Jenny, A. (2011) Planar cell polarity in Drosophila. Organogenesis, 7, 165-179.

4. Montcouquiol, M., Crenshaw, E.B. 3rd and Kelley, M.W. (2006) Noncanonical Wnt signaling and neural polarity. Annu. Rev. Neurosci., 29, $363-386$.

5. Tada, M. and Heisenberg, C.-P. (2012) Convergent extension: using collective cell migration and cell intercalation to shape embryos. Dev. Camb. Engl., 139, 3897-3904.

6. Wang, Y. and Nathans, J. (2007) Tissue/planar cell polarity in vertebrates: new insights and new questions. Dev. Camb. Engl., 134, 647-658.

7. Roszko, I., Sawada, A. and Solnica-Krezel, L. (2009) Regulation of convergence and extension movements during vertebrate gastrulation by the Wnt/PCP pathway. Semin. Cell Dev. Biol., 20, 986-997.

8. Wallingford, J.B. (2005) Neural tube closure and neural tube defects: studies in animal models reveal known knowns and known unknowns. Am. J. Med. Genet. C Semin. Med. Genet., 135C, 59-68.

9. Bassuk, A.G. and Kibar, Z. (2009) Genetic basis of neural tube defects. Semin. Pediatr. Neurol., 16, 101-110.

10. Wallingford, J.B., Niswander, L.A., Shaw, G.M. and Finnell, R.H. (2013) The continuing challenge of understanding, preventing, and treating neural tube defects. Science, 339, 1222002.

11. Juriloff, D.M. and Harris, M.J. (2012) A consideration of the evidence that genetic defects in planar cell polarity contribute to the etiology of human neural tube defects. Birt. Defects Res. A. Clin. Mol. Teratol., 94, 824-840.

12. Rossi, A., Biancheri, R., Cama, A., Piatelli, G., Ravegnani, M. and Tortori-Donati, P. (2004) Imaging in spine and spinal cord malformations. Eur. J. Radiol., 50, 177-200

13. Peradziryi, H., Tolwinski, N.S. and Borchers, A. (2012) The many roles of PTK7: a versatile regulator of cell-cell communication. Arch. Biochem. Biophys., 524, 71-76.

14. Schwarz-Romond, T., Asbrand, C., Bakkers, J., Kühl, M., Schaeffer, H.-J., Huelsken, J., Behrens, J., Hammerschmidt, M. and Birchmeier, W. (2002) The ankyrin repeat protein Diversin recruits Casein kinase Iepsilon to the beta-catenin degradation complex and acts in both canonical Wnt and Wnt JNK signaling. Genes Dev., 16, 2073-2084.

15. Li, S., Esterberg, R., Lachance, V., Ren, D., Radde-Gallwitz, K., Chi, F., Parent, J.-L., Fritz, A. and Chen, P. (2011) Rack1 is required for Vangl2 membrane localization and planar cell polarity signaling while attenuating canonical Wnt activity. Proc. Natl. Acad. Sci. U. S. A., 108, 2264-2269.

16. Bryja, V., Andersson, E.R., Schambony, A., Esner, M., Bryjová, L., Biris, K.K., Hall, A.C., Kraft, B., Cajanek, L., Yamaguchi, T.P. et al. (2009) The extracellular domain of Lrp5/6 inhibits noncanonical Wnt signaling in vivo. Mol. Biol. Cell, 20, 924-936.

17. Tahinci, E., Thorne, C.A., Franklin, J.L., Salic, A., Christian, K.M., Lee, L.A., Coffey, R.J. and Lee, E. (2007) Lrp6 is required for convergent extension during Xenopus gastrulation. Dev. Camb. Engl., 134, 4095-4106.

18. He, X., Semenov, M., Tamai, K. and Zeng, X. (2004) LDL receptor-related proteins 5 and 6 in Wnt/beta-catenin signaling: arrows point the way. Dev. Camb. Engl., 131, 1663-1677.

19. Pinson, K.I., Brennan, J., Monkley, S., Avery, B.J. and Skarnes, W.C. (2000) An LDL-receptor-related protein mediates Wnt signalling in mice. Nature, 407, 535-538.
20. Zhou, C.J., Wang, Y.-Z., Yamagami, T., Zhao, T., Song, L. and Wang, K. (2010) Generation of Lrp6 conditional gene-targeting mouse line for modeling and dissecting multiple birth defects/congenital anomalies. Dev. Dyn. Off. Publ. Am. Assoc. Anat., 239, 318-326.

21. Kokubu, C., Heinzmann, U., Kokubu, T., Sakai, N., Kubota, T., Kawai, M., Wahl, M.B., Galceran, J., Grosschedl, R., Ozono, K. et al. (2004) Skeletal defects in ringelschwanz mutant mice reveal that Lrp6 is required for proper somitogenesis and osteogenesis. Dev. Camb. Engl., 131, 5469-5480.

22. Fossat, N., Jones, V., Khoo, P.-L., Bogani, D., Hardy, A., Steiner, K., Mukhopadhyay, M., Westphal, H., Nolan, P.M., Arkell, R. et al. (2011) Stringent requirement of a proper level of canonical WNT signalling activity for head formation in mouse embryo. Dev. Camb. Engl., 138, 667-676.

23. Carter, M., Chen, X., Slowinska, B., Minnerath, S., Glickstein, S., Shi, L., Campagne, F., Weinstein, H. and Ross, M.E. (2005)Crooked tail(Cd) model of human folate-responsive neural tube defects is mutated in Wnt coreceptor lipoprotein receptor-related protein 6. Proc. Natl. Acad. Sci. U. S. A., 102, $12843-12848$.

24. Hentges, K.E., Nakamura, H., Furuta, Y., Yu, Y., Thompson, D.M., O'Brien, W., Bradley, A. and Justice, M.J. (2006) Novel lethal mouse mutants produced in balancer chromosome screens. Gene Expr. Patterns GEP, $\mathbf{6}$, $653-665$.

25. Kibar,Z., Vogan, K.J., Groulx, N., Justice, M.J., Underhill, D.A. and Gros, P. (2001) Ltap, a mammalian homolog of Drosophila Strabismus/Van Gogh, is altered in the mouse neural tube mutant Loop-tail. Nat. Genet., 28, 251-255.

26. Ezan, J. and Montcouquiol, M. (2013) Revisiting planar cell polarity in the inner ear. Semin. Cell Dev. Biol., 24, 499-506.

27. Montcouquiol, M., Rachel, R.A., Lanford, P.J., Copeland, N.G., Jenkins, N.A. and Kelley, M.W. (2003) Identification of Vangl2 and Scrb1 as planar polarity genes in mammals. Nature, $\mathbf{4 2 3}, 173-177$.

28. McNeill, H. (2010) Planar cell polarity: keeping hairs straight is not so simple. Cold Spring Harb. Perspect. Biol., 2, a003376.

29. Diebler, C. and Dulac, O. (1983) Cephaloceles: clinical and neuroradiological appearance. associated cerebral malformations. Neuroradiology, 25, 199-216.

30. Mao, B., Wu, W., Li, Y., Hoppe, D., Stannek, P., Glinka, A. and Niehrs, C. (2001) LDL-receptor-related protein 6 is a receptor for Dickkopf proteins. Nature, 411, 321-325.

31. Itasaki, N., Jones, C.M., Mercurio, S., Rowe, A., Domingos, P.M., Smith, J.C. and Krumlauf, R. (2003) Wise, a context-dependent activator and inhibitor of Wnt signalling. Dev. Camb. Engl., 130, 4295-4305.

32. Caneparo, L., Huang, Y.-L., Staudt, N., Tada, M., Ahrendt, R., Kazanskaya, O., Niehrs, C. and Houart, C. (2007) Dickkopf-1 regulates gastrulation movements by coordinated modulation of Wnt/beta catenin and Wnt/PCP activities, through interaction with the Dally-like homolog Knypek. Genes Dev, 21, 465-480.

33. Gray, J.D., Kholmanskikh, S., Castaldo, B.S., Hansler, A., Chung, H., Klotz, B., Singh, S., Brown, A.M.C. and Ross, M.E. (2013) LRP6 Exerts non-canonical effects on Wnt signaling during neural tube closure. Hum. Mol. Genet., 22, 4267-4281.

34. Murdoch, J.N., Henderson, D.J., Doudney, K., Gaston-Massuet, C., Phillips, H.M., Paternotte, C., Arkell, R., Stanier, P. and Copp, A.J. (2003) Disruption of scribble (Scrb1) causes severe neural tube defects in the circletail mouse. Hum. Mol. Genet., 12, 87-98.

35. Etheridge, S.L., Ray, S., Li, S., Hamblet, N.S., Lijam, N., Tsang, M., Greer, J., Kardos, N., Wang, J., Sussman, D.J. et al. (2008) Murine dishevelled 3 functions in redundant pathways with dishevelled 1 and 2 in normal cardiac outflow tract, cochlea, and neural tube development. PLoS Genet., 4, e1000259.

36. Lu, X., Borchers, A.G.M., Jolicoeur, C., Rayburn, H., Baker, J.C. and Tessier-Lavigne, M. (2004) PTK7/CCK-4 is a novel regulator of planar cell polarity in vertebrates. Nature, $\mathbf{4 3 0}, 93-98$.

37. Kibar,Z., Torban, E., McDearmid, J.R., Reynolds, A., Berghout, J., Mathieu, M., Kirillova, I., De Marco, P., Merello, E., Hayes, J.M. et al. (2007) Mutations in VANGL1 associated with neural-tube defects. N. Engl. J. Med., 356, 1432-1437.

38. Bosoi, C.M., Capra, V., Allache, R., Trinh, V.Q.-H., De Marco, P., Merello, E., Drapeau, P., Bassuk, A.G. and Kibar, Z. (2011) Identification and characterization of novel rare mutations in the planar cell polarity gene PRICKLE1 in human neural tube defects. Hum. Mutat., 32, 1371-1375.

39. De Marco, P., Merello, E., Rossi, A., Piatelli, G., Cama, A., Kibar, Z. and Capra, V. (2012) FZD6 is a novel gene for human neural tube defects. Hum. Mutat., 33, 384-390 
40. Seo, J.H., Zilber, Y., Babayeva, S., Liu, J., Kyriakopoulos, P., De Marco, P., Merello, E., Capra, V., Gros, P. and Torban, E. (2011) Mutations in the planar cell polarity gene, Fuzzy, are associated with neural tube defects in humans. Hum. Mol. Genet., 20, 4324-4333.

41. Allache, R., De Marco, P., Merello, E., Capra, V. and Kibar, Z. (2012) Role of the planar cell polarity gene CELSR1 in neural tube defects and caudal agenesis. Birt. Defects Res. A. Clin. Mol. Teratol., 94, $176-181$.

42. Robinson, A., Escuin, S., Doudney, K., Vekemans, M., Stevenson, R.E., Greene, N.D.E., Copp, A.J. and Stanier, P. (2012) Mutations in the planar cell polarity genes CELSR1 and SCRIB are associated with the severe neural tube defect craniorachischisis. Hum. Mutat., 33, 440-447.

43. Weidinger, G. and Moon, R.T. (2003) When Wnts antagonize Wnts. J. Cell Biol., 162, 753-755.

44. Westfall, T.A., Brimeyer, R., Twedt, J., Gladon, J., Olberding, A., Furutani-Seiki, M. and Slusarski, D.C. (2003) Wnt-5/pipetail functions in vertebrate axis formation as a negative regulator of $\mathrm{Wnt} /$ beta-catenin activity. J. Cell Biol., 162, 889-898.
45. Andersson, E.R., Bryjova, L., Biris, K., Yamaguchi, T.P., Arenas, E. and Bryja, V. (2010) Genetic interaction between Lrp6 and Wnt5a during mouse development. Dev. Dyn. Off. Publ. Am. Assoc. Anat., 239, 237-245.

46. Alarcón, M.A., Medina, M.A., Hu, Q., Avila, M.E., Bustos, B.I., Pérez-Palma, E., Peralta, A., Salazar, P., Ugarte, G.D., Reyes, A.E. et al. (2013) A novel functional low-density lipoprotein receptor-related protein 6 gene alternative splice variant is associated with Alzheimer's disease. Neurobiol. Aging, 34, 1709.e9-18.

47. Mani, A., Radhakrishnan, J., Wang, H., Mani, A., Mani, M.-A., Nelson-Williams, C., Carew, K.S., Mane, S., Najmabadi, H., Wu, D. et al. (2007) LRP6 Mutation in a family with early coronary disease and metabolic risk factors. Science, 315, 1278-1282.

48. Guyot, M.-C., Bosoi, C.M., Kharfallah, F., Reynolds, A., Drapeau, P., Justice, M., Gros, P. and Kibar, Z. (2011) A novel hypomorphic Looptail allele at the planar cell polarity Vangl2 gene. Dev. Dyn. Off. Publ. Am. Assoc. Anat., 240, 839-849.

49. Li, M.Z. and Elledge, S.J. (2007) Harnessing homologous recombination in vitro to generate recombinant DNA via SLIC. Nat. Methods, 4, 251-256. 